\title{
Preface to CLEI 2011 special issue
}

\author{
José Aguilar \\ Universidad de Los Andes, CEMISID Mérida, \\ Venezuela \\ John Atkinson \\ Universidad de Concepción \\ Chile \\ Yezid Donoso \\ Universidad del Norte \\ Colombia \\ David Fernández-Baca \\ Iowa State University \\ USA \\ Ernst L. Leiss \\ Department of Computer Science, University of Houston, \\ USA \\ Gabriela Marin \\ Escuela de Ciencias de la Computacion e Informatica, Universidad de Costa Rica \\ Costa Rica \\ Luciana Nedel \\ Federal University of Rio Grande do Sul \\ Brazil \\ Oscar Pastor \\ Universitat Politècnica de València \\ Spain
}

This special issue contains papers from CLEI 2011 which took place in October 2011 in Quito, Ecuador. The editors for this special issue were the chairs of the six symposia that constituted the conference CLEI 2011, plus the general chair of the program committee.

As every year, the program committee of the conference carried out a rigorous evaluation process which resulted in the selection of papers to be presented at the conference and published in the conference proceedings. In addition, the program committee of the conference singled out a number of papers that were considered especially meritorious.

In 2011, this process was bifurcated inasmuch as meritorious papers with a theoretical content were published in Elsevier's ENTCS (without any change from the conference papers) while the more applied papers underwent the customary evaluation process for papers to be included in a special issue of CLEI ej, namely the "Best Papers from CLEI 2011" issue. In total, 18 papers of applied content were determined to be meritorious and were invited to be considered for the special issue. These papers had to be extended versions of the conference paper and had to be in English. There was an additional refereeing process of those papers that were submitted by their authors.

The final outcome was that eight papers were selected; these are the papers contained in the special issue you are reading. We hope you find these papers interesting and worth your while studying.

With best regards,

Jose Aguilar John Atkinson

Yezid Donoso

David Fernández-Baca

Ernst L. Leiss Gabriela Marín

Luciana Nedel

Oscar Pastor 